\title{
L'intervention tarifaire du Conseil fédéral aura un large impact
}

\section{Christian Oeschger}

Collaborateur scientifique, division Médecine et tarifs ambulatoires

Le Conseil fédéral intervient une deuxième fois dans la structure tarifaire TARMED. Alors que la consultation est en cours, les premières analyses de la FMH révèlent une image sombre: les pertes s'avèrent en partie importantes, avant tout dans le domaine opératoire et invasif mais d'autres disciplines (spécialistes) seront aussi fortement concernées. La FMH s'oppose à des interventions isolées et, dans le cadre de son projet TARCO, elle continue de poursuivre l'objectif d'une révision globale.

Le $1^{\text {er }}$ janvier 2018, le Conseil fédéral interviendra une deuxième fois dans la structure tarifaire TARMED. A l'occasion de sa conférence de presse tenue à Berne le 22 mars 2017, il a mis en consultation son projet d'ordonnance. Le Conseil fédéral prévoit une intervention beaucoup plus conséquente qu'en 2014. Cette deuxième intervention aura un impact financier important sur les cabinets médicaux et les centres ambulatoires hospitaliers. En plus d'introduire des règles de facturation supplémentaires, le Conseil fédéral planifie des interventions directes sur la tarification. La FMH a publié tous les documents de la consultation sur son site Internet: www.fmh.ch $\rightarrow$ Tarifs ambulatoires $\rightarrow$ TARMED Tarif $\rightarrow$ Modifications du TARMED à partir du $1^{\text {er }}$ janvier 2018.

\section{Les mesures de l'ordonnance en détail}

Les mesures que propose l'Office fédéral de la santé publique (OFSP) au Conseil fédéral sont vastes et complexes. Elles se basent sur le tarif actuellement en vigueur qui avait déjà subi une perte de $8,5 \%$ dans plusieurs cha-

\footnotetext{
Journée des délégués tarifaires

La Journée des délégués tarifaires de la FMH qui s'est tenue le 20 avril 2017 à Berne a une nouvelle fois suscité un grand intérêt de la part des participants, plus de 80 présidents et délégués tarifaires des organisations représentées à la Chambre médicale. Les délégués tarifaires trouveront dès à présent les présentations du Dr Urs Stoffel, de la Dresse Susanne Christen, de Kerstin Schutz, Sabine Zehnder, Thomas Kessler et Christian Oeschger sur myFMH, dans le dossier Tarifdelegierten-Tag - Journée des délégués tarifaires $\rightarrow$ 20.04.2017.
}

pitres en 2014 et comportent des baisses supplémentaires.

L'OFSP propose différentes catégories d'adaptation. Nous abordons ci-dessous les points les plus importants pour le corps médical:

Les mesures de la première catégorie prévoient entre autres un nivellement de la «valeur intrinsèque quantitative». Variant aujourd'hui entre 0,905 (FMH5) et 2,2625 (FMH12), elle doit être uniformisée à 0,968 dans tout le tarif. Aux côtés du revenu de référence, du temps de travail annuel et de la "productivité médicale», elle est décisive pour le point tarifaire de la "prestation médicale (PM)». Avec cette mesure, les prestations dotées d'une "valeur intrinsèque quantitative» de FMH- 5 bénéficieront d'une légère amélioration tandis que des pertes importantes sont attendues pour toutes les autres. Le "médecin praticien" au bénéfice d'une formation postgrade de trois ans sera même rabaissé à 0,940 .

Par ailleurs, le Conseil fédéral entend serrer la vis concernant la "productivité médicale» qui doit être supprimée pour toutes les prestations opératoires. Cette mesure a aussi un impact (en partie important) sur la prestation médicale et la diminue un peu plus. Le Conseil fédéral intervient également sur la "prestation technique (PT)» en réduisant de $10 \%$ le barème de coûts de plusieurs unités fonctionnelles et du même coup aussi le point tarifaire de la prestation technique. Plusieurs domaines spécifiques du tarif enregistreront des réductions conséquentes. Pour le chapitre de l'imagerie médicale, l'OFSP propose de supprimer entièrement la prestation médicale des examens IRM et CT et de ne rémunérer le médecin que pour l'interprétation 
des scanners et des IRM. Les propositions de l'OFSP prévoient également de supprimer entièrement des prestations ou de réduire fortement les minutages des positions tarifaires relatives à l'opération de la cataracte, aux injections intravitréennes, aux ECG d'effort et Holter, à la coloscopie et à la radiothérapie stéréotaxique.

La deuxième catégorie de mesures se distingue par l'introduction de limitations dans les règles de facturation. L'OFSP propose de convertir certaines prestations à l'acte des examens par le spécialiste en prestations au temps et de limiter le volume (facturable) aussi bien du point de vue du nombre de séances que de l'intervalle pendant lequel ces séances peuvent être facturées. Un principe similaire est appliqué aux prestations en l'absence du patient: si leur interprétation est désormais plus précise, ces prestations sont également limitées. La troisième catégorie de mesures concerne la suppression du supplément de $10 \%$ sur le matériel à usage courant et les implants (Interprétation générale 20) et la suppression de positions tarifaires pour les prestations hospitalières et pour les prestations de l'assuranceaccidents, de l'assurance-invalidité ou de l'assurance militaire, ainsi que de tous les renvois à la loi.

De plus amples informations relatives aux différentes mesures sont accessibles par les membres sur myFMH: https://myfmh.fmh.ch/ $\rightarrow$ Vernehmlassung Tarifeingriff - Consultation intervention tarifaire.

\section{Impact massif sur la "prestation médicale»}

La FMH ne pourra rendre une évaluation complète et détaillée des mesures et de leur impact qu'après des analyses supplémentaires en interne et après simulations. Celles-ci seront communiquées en temps utile.

Mais il est aujourd'hui certain que ce vaste paquet de mesures aura un impact financier en partie important pour les membres de la FMH. Rien que les effets sur la "valeur intrinsèque quantitative» seront déjà conséquents dans plusieurs disciplines. A cela s'ajoutent la hausse de la productivité médicale pour l'ensemble des opérations, les corrections concrètes du minutage de certaines positions tarifaires et les nouvelles limitations prévues sur quelques-unes des principales prestations au temps.

Les chiffres de la FMH et de la Caisse des médecins montrent que d'importantes hausses ont impacté le travail salarié non médical sans qu'aucune adaptation du tarif n'ait eu lieu depuis l'introduction du TARMED. En parallèle, les progrès techniques et les évolutions démographiques se traduisent par un besoin en person- nel plus élevé dans les cabinets médicaux. Par ailleurs, les médecins hospitaliers ont enregistré une hausse de leur salaire depuis 2014. Pour que le passage à la pratique privée reste attractif après les années de spécialisation à l'hôpital, les futurs médecins installés doivent avoir aussi la possibilité de générer des revenus concurrentiels. Avec les économies unilatérales telles que les propose l'OFSP, ce n'est plus possible.

\section{Une révision globale plutôt que des interventions ponctuelles}

Ni la FMH ni les organisations représentées à la Chambre médicale ne contestent la nécessité d'actualiser une structure tarifaire ambulatoire introduite sur tout le territoire en 2004 et, de ce fait, dépassée. En revanche, la FMH s'oppose à toute intervention ponctuelle qui ne ferait qu'engendrer de nouvelles distorsions de la structure tarifaire actuellement en vigueur. Après la votation générale de mai 2016, la FMH a lancé le projet de révision TARCO en vue d'améliorer sa première proposition de révision. TARCO vise une tarification appropriée et conforme aux règles applicables en économie d'entreprise de toutes les prestations médicales ambulatoires. Pour le corps médical, une révision tarifaire globale en concertation avec toutes les organisations médicales et tous les partenaires tarifaires constitue la seule voie pertinente à suivre.

\section{Profitez de vos droits}

Même si vous n'avez pas été convié à la consultation, vous pouvez vous prononcer sur l'ordonnance d'ici le 21 juillet 2017. Toutes les sociétés de discipline sont invitées à faire usage de ce droit et à déposer leur prise de position par voie électronique ou courrier postal à I'adresse suivante:

Office fédéral de la santé publique (OFSP)

Unité de direction Assurance maladie et accident

Division Prestations

3003 Berne

abteilung-leistungen[at]bag.admin.ch

Tél. +4158462 2111

Nous vous serions reconnaissants de joindre une copie de votre prise de position à la FMH afin que vos arguments puissent être inclus dans notre réponse consolidée. Pour que nous disposions de suffisamment de temps, votre prise de position devrait nous parvenir au plus tard d'ici le $1^{\text {er }}$ juin 2017 à I'adresse suivante:

$\mathrm{FMH}$

Division Médecine et tarifs ambulatoires

Frohburgstrasse 15

4600 Olten

tarife.ambulant[at]fmh.ch

Tél. +41313501230 


\section{Calendrier}

\begin{tabular}{ll}
\hline 22.03.2017 & Ouverture de la consultation \\
\hline $\begin{array}{ll}\text { 29.03.2017 } & \text { Publication de la première appréciation } \\
\text { de la FMH }\end{array}$ \\
\hline 17.04 .2017 & Analyse approfondie de la FMH à l'interne \\
21.06 .2017 & Analyse approfondie de la FMH pour les \\
\hline $15.05 .2017-$ & sociétés de discipline et cantonales affiliées \\
\hline 21.06 .2017 & $\begin{array}{l}\text { permettre aux membres de mesurer l'impact } \\
\text { de l'intervention }\end{array}$ \\
\hline 01.06 .2017 & Délai d'envoi des prises de position des \\
& sociétés de discipline à la FMH \\
\hline 21.06 .2017 & $\begin{array}{l}\text { Séance extraordinaire du Comité central } \\
\text { de la FMH pour définir la prise de position } \\
\text { de la FMH sur l'intervention tarifaire }\end{array}$ \\
\hline 01.01 .2018 & Fin de la consultation \\
\hline
\end{tabular}

\section{Nous vous assistons vous en particulier et votre société de discipline}

Le département Médecine et tarifs ambulatoires de la FMH et NewIndex vous aident volontiers à réaliser les analyses nécessaires à votre prise de position à l'attention de l'Office fédéral de la santé publique. Vous pouvez nous contacter à tarife.ambulant[at]fmh.ch ou info[at]newindex.ch. Nous pouvons vous transmettre une appréciation détaillée du projet d'ordonnance sur la base de la structure des volumes de votre société de discipline ou de votre société cantonale de médecine. Nous conseillons aussi les médecins en particulier. Vous trouverez des informations à ce sujet dans les prochaines semaines sur notre site Internet. 